\title{
Effet améliorateur d'un apport extérieur de calcium et de potassium sous différentes formes sur la tolérance à la salinité de l'amarante (Amaranthus cruentus L.)
}

\author{
Richard Atou¹, Eunice Henry1, Armel C. G. Mensah², Baudouin G. Gouveitcha1', Belvida Loko', \\ Agapit D. Wouyou ${ }^{1}$, Françoise Assogba Komlan ${ }^{2}$ et Christophe Bernard Gandonou ${ }^{1 *}$ \\ 1 Unité de Recherche sur l'Adaptation des Plantes aux Stress Abiotiques, les Métabolites Secondaires et \\ l'Amélioration des Productions Végétales, Laboratoire de Physiologie Végétale et d'Etude des Stress \\ Environnementaux, Faculté des Sciences et Techniques (FAST/UAC), 01BP526, Tri Postal, Cotonou, République du \\ Bénin. \\ ${ }^{2}$ Centre de Recherches Agricoles Plantes Pérennes (CRA-PP), Pobè, Institut National des Recherches Agricoles du \\ Bénin (INRAB), Abomey-Calavi, Bénin \\ Auteur pour correspondance : Courriel : ganchrist@hotmail.com, Tél. : (00229) 97396978
}

Original submitted in on $18^{\text {th }}$ November 2019. Published online at www.m.elewa.org/journals/ on $29^{\text {th }}$ February 2020 https://doi.org/10.35759/JABs.v146.5

\section{RÉSUMÉ}

Objectifs : La salinité est un facteur abiotique qui réduit la production agricole. Cette étude a évalué l'effet améliorateur d'une application exogène de potassium et de calcium sous différentes formes sur la croissance des plantes d'amarante cultivés sous stress salin et l'implication de l'accumulation des ions sodium et potassium dans cette amélioration.

Méthodologie et résultats: Les jeunes plantes ont été soumises à dix traitements comprenant le témoin sans $\mathrm{NaCl} ; 120 \mathrm{mM}$ de $\mathrm{NaCl}$ et une combinaison entre $120 \mathrm{mM}$ de $\mathrm{NaCl}$ et un apport de 40 ou 60 $\mathrm{mM}$ de $\mathrm{CaSO}_{4} ; \mathrm{CaCl}_{2} ; \mathrm{KNO}_{3}$ ou $\mathrm{K}_{2} \mathrm{SO}_{4}$. La croissance des plantes ainsi que les teneurs en sodium et potassium des feuilles et racines ont été déterminées après deux semaines. Les résultats indiquent que l'apport extérieur des différents composés a atténué les effets néfastes du $\mathrm{NaCl}$ sur la croissance des plantes à travers une exclusion du sodium des feuilles et une meilleure accumulation du potassium.

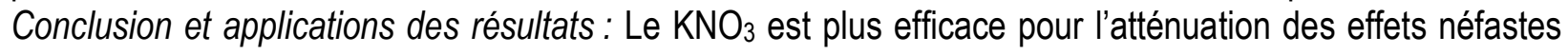
du $\mathrm{NaCl}$ sur la croissance des plantes d'amarante, suivi par le $\mathrm{CaSO}_{4}$. Cette atténuation est due à une meilleure exclusion du $\mathrm{Na}^{+}$des feuilles associée à une meilleure accumulation de $\mathrm{K}^{+}$conduisant à un meilleur rapport $\mathrm{K}^{+} / \mathrm{Na}^{+}$. Les deux composés pourront être conseillés aux producteurs d'amarante des zones côtières du Bénin affectées par la salinité.

Mots clés : Amaranthus cruentus, "Fôtêtê », $\mathrm{NaCl}$, atténuation des effets de la salinité, $\mathrm{CaCl}_{2}, \mathrm{CaSO}_{4}$, $\mathrm{KNO}_{3}, \mathrm{~K}_{2} \mathrm{SO}_{4}$. 
Ameliorative effect of exogenous application of different forms of calcium and potassium on amaranth (Amaranthus cruentus L.) salt tolerance

\section{ABSTRACT}

Objectives: Salinity is an abiotic factor that reduces agricultural production al around the world. This study aims to evaluate the ameliorative effect of an exogenous application of potassium and calcium in different forms on the growth of amaranth plants grown under salt stress and the implication of sodium and potassium ions accumulation in this improvement.

Methodology and results: Young plants were subjected to ten treatments including the control without $\mathrm{NaCl}$; $120 \mathrm{mM} \mathrm{NaCl}$ and a combination between $120 \mathrm{mM} \mathrm{NaCl}$ and exogenous application of 40 or $60 \mathrm{mM}$ $\mathrm{CaSO}_{4} ; \mathrm{CaCl}_{2} ; \mathrm{KNO}_{3}$ or $\mathrm{K}_{2} \mathrm{SO}_{4}$. Plants growth as well as sodium and potassium ions contents were determined in leaves and roots after two weeks. Results indicate that exogenous application of these compounds alleviates the detrimental effects of $\mathrm{NaCl}$ on amaranth plant growth through sodium exclusion from leaves and better potassium accumulation.

Conclusion and applications of results: $\mathrm{KNO}_{3}$ is the most effective compounds in alleviating of $\mathrm{NaCl}$ detrimental effect on amaranth plant growth, followed by $\mathrm{CaSO}_{4}$. This growth improvement was due to better $\mathrm{Na}^{+}$exclusion from leaves associated with better $\mathrm{K}^{+}$accumulation resulting in a best $\mathrm{K}^{+} / \mathrm{Na}^{+}$ratio. Both compounds may be recommended to amaranth producers in coastal areas of Benin affected by salinity.

Key words: Amaranthus cruentus, "Fôtêtê », $\mathrm{NaCl}$, alleviation of salinity effects, $\mathrm{CaCl}_{2}, \mathrm{CaSO}_{4}$, $\mathrm{KNO}_{3}, \mathrm{~K}_{2} \mathrm{SO}_{4}$.

\author{
Abbréviations \\ $\mathrm{T}=0 \mathrm{mM} \mathrm{NaCl}$ \\ $\mathrm{S}=120 \mathrm{mM} \mathrm{NaCl}$ \\ $\mathrm{S}+\mathrm{CaSO}_{4}=120 \mathrm{mM} \mathrm{NaCl}+40$ or $60 \mathrm{mM} \mathrm{CaSO} 4$ \\ $\mathrm{S}+\mathrm{CaCl}_{2}=120 \mathrm{mM} \mathrm{NaCl}+40$ or $60 \mathrm{mM} \mathrm{CaCl} 2$ \\ $\mathrm{S}+\mathrm{KNO}_{3}=120 \mathrm{mM} \mathrm{NaCl}+40$ or $60 \mathrm{mM} \mathrm{KNO}_{3}$ \\ $\mathrm{S}+\mathrm{K}_{2} \mathrm{SO}_{4}=120 \mathrm{mM} \mathrm{NaCl}+40$ or $60 \mathrm{mM} \mathrm{K}_{2} \mathrm{SO}_{4}$
}

\section{INTRODUCTION}

L'amarante (Amaranthus spp.) est considérée comme une culture prometteuse pour les terres marginales et les régions semi-arides en raison de sa haute valeur nutritive et de sa grande adaptabilité à divers environnements (Cunningham et al., 1992 ; Allemann et al., 1996) où le problème de la salinité est aigu (Bhattacharjee, 2008). C'est le principal légume-feuille au Bénin où elle est cultivée dans le sud, dans les zones urbaines et péri-urbaines et dans la vallée de l'Ouémé (Adorgloh-Hessou, 2006). Dans certaines de ces zones notamment côtières, les eaux utilisées pour l'irrigation contiennent généralement des teneurs en sels qui peuvent inhiber la croissance des plantes et réduire le rendement des cultures (Gandonou et Abou, 2018). Plusieurs études antérieures ont montré que la salinité réduit la croissance chez diverses espèces d'amarante (Makus, 2003 ; Omami, 2005 ; Omami et Hammes, 2006; Qin et al., 2013 ; Amukali et al., 2015; Lavini et al., 2016). Au Bénin, il a été rapporté que la croissance du cultivar locale le plus largement prisé et produit au Bénin est réduite par des concentrations de $\mathrm{NaCl}$ aussi faibles que $30 \mathrm{mM}$ de $\mathrm{NaCl}$ (Wouyou et al., 2017). Par ailleurs, plusieurs travaux ont révélé que la réduction de la croissance des plantes d'amarante sous l'effet de la salinité est principalement la conséquence de l'accumulation d'ions devenant toxiques $\left(\mathrm{Na}^{+}\right.$et $\mathrm{Cl}^{-}$ ) pour les activités métaboliques de la plante (Omami, 2005 ; Odjegba et Chukwunwike., 2012). En effet, ces ions peuvent perturber la capacité de 
la plante à contrôler l'accumulation d'autres ions importants (Wouyou et al., 2019). Parmi ces ions, l'ion sodium $\left(\mathrm{Na}^{+}\right)$occupe une place de choix. En effet, il est globalement admis chez la plupart des espèces végétales que le $\mathrm{Na}^{+}$est accumulé à des doses toxiques avant le $\mathrm{Cl}$ - (Negrão et al., 2017) et que le $\mathrm{Cl}^{-}$est considéré comme étant moins toxique que le $\mathrm{Na}^{+}$(Munns et tester, 2008). L'amélioration de la tolérance d'une plante à la salinité peut être obtenue, pas seulement à travers la sélection génétique, mais également à travers l'utilisation d'outils physiologiques adaptés (Manaa et al., 2014). Ainsi, l'une des approches pour limiter les effets de la salinité sur les plantes consiste à procéder à un enrichissement des teneurs en nutriments du substrat utilisé pour la culture des plantes à travers l'apport extérieur de composés tels que l'azote $(\mathrm{N})$, le phosphore $(\mathrm{P})$, le potassium $(\mathrm{K})$, le magnésium $(\mathrm{Mg})$ et le calcium (Ca) dans le but de réduire les effets négatifs $d u$ $\mathrm{Na}^{+}$et du Cl- sur les plantes (Kaya et al., 2002; Song et Roe, 2008). Un intérêt particulier a été porté sur le calcium du fait de son habilité à induire un effet protecteur des plantes en conditions environnementales défavorables (Manaa et al., 2014). Le calcium joue un rôle vital dans la tolérance au stress salin puisqu'il induit les activités des enzymes anti-oxydantes et réduit la peroxydation des lipides des membranes cellulaires sous stress abiotiques (Jiang et Huang, 2001 ; Khan et al., 2010). De nombreuses études ont suggéré que le $\mathrm{Na}^{+}$entre en compétition avec le $\mathrm{Ca}^{2+}$ au niveau des sites de fixation en condition de stress salin et que le $\mathrm{Ca}^{2+}$ apoplastique supprime directement les symptômes produits par les toxicités minérales. L'effet améliorateur $\mathrm{du}^{\mathrm{Ca}}{ }^{2+}$

\section{MATERIEL ET METHODES}

Matériel végétal: II est constitué par le cultivar d'amarante dénommé 'Locale' communément appelé " Fôtêtê » en langue Fongbé. Les semences ont été fournies par le Sous-Programme 'Cultures Maraîchères' de I'Institut National des Recherches Agricoles du Bénin (INRAB).

\section{Méthodologie}

Conditions expérimentales: L'expérience a été réalisée dans une serre à l'Institut National des externe chez les plantes exposées à la salinité peut être associé au maintien d'un rapport $\mathrm{K}+\mathrm{Na}^{+}$ optimal et à une homéostasie dans le cytosol en relation avec une inhibition de l'influx de $\mathrm{Na}^{+}$(entrée dans la cellule), de l'efflux de $\mathrm{K}^{+}$(sortie de la cellule) ou la promotion de l'efflux de $\mathrm{Na}^{+}$ (sortie de la cellule) et l'influx de $\mathrm{K}^{+}$(entrée dans la cellule) à travers la membrane plasmique (Elphick et al., 2001; Demidchik et Tester, 2002; Shabala et al., 2006). De même, plusieurs études ont montré que l'application extérieure de potassium (K) sous différentes formes améliore la tolérance à la salinité en induisant notamment une exclusion $\mathrm{du} \mathrm{Na}^{+}$et une accumulation de $\mathrm{K}^{+}$. C'est le cas chez le blé (Hussain et al., 2013) et l'arachide (Chakraborty et al., 2016). Chez l'amarante, à part les travaux de Omami et Hammes (2006) qui ont évalué l'effet améliorateur d'un apport extérieur de calcium sous les formes de $\mathrm{CaCl}_{2}$ et $\mathrm{CaSO}_{4}$ sur la croissance et l'absorption des ions et qui ont montré que le sulfate de calcium $\left(\mathrm{CaSO}_{4}\right)$ était le plus efficace, pratiquement aucune autre étude ne s'est intéressée à l'amélioration de la tolérance de l'amarante à la salinité à travers l'apport extérieur de composés minéraux. Dans la présente étude, nous avons évalué l'effet d'un apport extérieur de potassium et de calcium sous différentes formes sur la croissance et la nutrition minérale de l'amarante cultivée sous stress salin. Le principal objectif est de mettre en évidence les améliorations de croissance qu'engendrerait un apport extérieur de ces composés et les mécanismes par lesquels ces composés provoquent cette amélioration de croissance notamment en relation avec l'accumulation des ions sodium et potassium.

Recherches Agricoles du Bénin (INRAB)/ (AbomeyCalavi, République du Bénin) de juillet à août 2018. Les plantes ont été cultivées à une température de 26 ${ }^{\circ} \mathrm{C} / 22$ jour / nuit avec de la lumière naturelle et une humidité relative de $55 \%$. Les graines ont été mises en germination dans des bacs remplies de terreau pendant deux semaines. Les jeunes plantes ont été ensuite transférées dans des petits pots en plastic de $5,8 \mathrm{~cm}$ de diamètre et de $6 \mathrm{~cm}$ de hauteur contenant un mélange 
de terreau et de sable 50:50 (une plante/pot) et cultivés pendant une semaine avant l'application du stress. Les plantes ont été soumises à un stress salin dans des grands pots de $11,3 \mathrm{~cm}$ de diamètre et de $14 \mathrm{~cm}$ de hauteur remplis avec $3 \mathrm{~kg}$ du même mélange. Les traitements ont consisté à l'arrosage des plantes tous les deux jours avec $100 \mathrm{ml} /$ pot de solution de $\mathrm{NaCl}$ seul ou en combinaison avec le $\mathrm{CaCl}_{2}$, le $\mathrm{CaSO}_{4}$, le $\mathrm{KNO}_{3}$ ou le $\mathrm{K}_{2} \mathrm{SO}_{4}$.

Dispositif expérimental: Le dispositif expérimental est de type factoriel à un seul facteur en randomisation complète. Le seul facteur représente les dix (10) traitements avec quatre répétitions. Ces traitements sont :

Témoin : $0 \mathrm{mM} \mathrm{NaCl}$

Traitement salin (S) : $120 \mathrm{mM} \mathrm{NaCl}$

Traitement salin avec application de $40 \mathrm{mM} \mathrm{CaSO}_{4}$ Traitement salin avec application de $60 \mathrm{mM} \mathrm{CaSO}_{4}$ Traitement salin avec application de $40 \mathrm{mM} \mathrm{CaCl}_{2}$ Traitement salin avec application de $60 \mathrm{mM} \mathrm{CaCl}_{2}$ Traitement salin avec application de $40 \mathrm{mM} \mathrm{KNO}_{3}$ Traitement salin avec application de $60 \mathrm{mM} \mathrm{KNO}_{3}$ Traitement salin avec application de $40 \mathrm{mM} \mathrm{K}_{2} \mathrm{SO}_{4}$ Traitement salin avec application de $60 \mathrm{mM} \mathrm{K}_{2} \mathrm{SO}_{4}$

Evaluation de la croissance : La hauteur des plantes et la longueur de la racine ont été mesurées. Les masses de matière fraîche de la partie aérienne et des racines ont été déterminées par pesée et les échantillons de chaque partie ont ensuite été transférés dans une étuve à $80^{\circ} \mathrm{C}$ pendant 72 heures pour déterminer les masses sèches. La surface foliaire a été obtenue par découpage des photocopies de trois feuilles bien développées de chaque traitement. Les mesures, les pesées et la photocopie des feuilles ont

\section{RESULTATS}

Effet des composés apportés sur la croissance des plants

Effet sur la croissance de la partie aérienne: Le stress salin a induit une réduction significative $(p=0,001)$ de la croissance relative de la hauteur de la plante $(\mathrm{CRH})$. L'apport des différents composés a induit une amélioration de la croissance en hauteur des plants uniquement pour les apports de $\mathrm{CaSO}_{4}, \mathrm{CaCl}_{2}$ et $\mathrm{KNO}_{3}$ de l'ordre de $39,18 \% ; 35,13 \%$ et $14,86 \%$ respectivement (fig. 1-A). Cependant, cette amélioration n'a été significative pour aucun de ces composés; une légère diminution a été même observée avec l'apport de $\mathrm{K}_{2} \mathrm{SO}_{4}$. Avec l'apport du calcium et du potassium à $60 \mathrm{mM}$ (fig. 1-B), une tendance similaire a été observée comme pour l'apport été faites avant l'application du stress salin (X0) ; ils ont été à nouveau faits 2 semaines après le traitement (X1). La croissance relative de chaque paramètre a été déterminé suivant la formule : $(\mathrm{X} 1-\mathrm{X} 0)$ / $\mathrm{X} 0$.

Extraction et estimation des concentrations d'ions : Pour la détermination des ions, les racines ont été rapidement rincées à l'eau distillée pour éliminer les ions fixés sur elles et ceux contenus dans l'apoplasme (Bourgeais-Chaillou et Guerrier, 1992). Les feuilles et les racines ont été individuellement séchées à l'étuve à $80^{\circ} \mathrm{C}$ pendant 72 heures et broyées dans un mortier, et la poudre a été séchée pendant 24 heures. Pour déterminer les concentrations de $\mathrm{Na}^{+}$et de $\mathrm{K}^{+}, 20 \mathrm{mg}$ des poudres sèches de feuille et de racine ont été placées dans des bocaux et digérées dans $1 \mathrm{ml}$ d'acide nitrique (68\%) à température ambiante. Après $24 \mathrm{~h}$, le volume a été complété à $20 \mathrm{ml}$ avec de l'eau distillée. Les solutions ont été filtrées avec du papier Whatman (85 mm, Grade 1). Le filtrat a été utilisé pour le dosage des ions grâce à un spectrophotomètre à flamme (Sherwood Model 360). Les quantités d'ions ont été exprimées en $\mathrm{mg} / \mathrm{g}$ de matière sèche (MS). Des solutions mères de $\mathrm{Na}^{+}$et de $\mathrm{K}^{+}$fournies avec le spectrophotomètre ont été utilisés comme standards.

Analyses statistiques: Pour tous les paramètres, les moyennes et erreurs standards ont été calculés avec trois (3) répétitions par traitement grâce au tableur Excel. Les résultats ont été soumis à l'analyse de la variance (ANOVA) à une (01) voie et les moyennes ont été comparées avec le Test de Tukey-Kramer. Les analyses ont été effectuées grâce au logiciel JMP Pro (JMP Pro SAS Institute, 2009).

de 40 mM. La croissance relative de la matière fraîche de la partie aérienne (CRMFPA) a subi une diminution significative $(p=0,001)$ sous l'effet de la salinité mais l'apport du calcium et du potassium sous différentes formes à $40 \mathrm{mM}$ a induit une amélioration de croissance significative $(\mathrm{p}=0,001)$ uniquement pour les apports de $\mathrm{CaSO}_{4}(111,68 \%) ; \mathrm{CaCl}_{2}(61,69 \%)$ et $\mathrm{KNO}_{3}$ (152,60\%) (fig. 2-A). . Avec l'apport du calcium et du potassium à $60 \mathrm{mM}$ (fig. 2-B), une tendance similaire a été observée comme pour l'apport de $40 \mathrm{mM}$ avec une amélioration significative de croissance $(p=0,001)$ uniquement pour les apports de $\mathrm{CaSO}_{4}$ et $\mathrm{KNO}_{3}$. Le stress salin a induit une diminution significative $(p=0,001)$ de la croissance de la matière sèche mais l'apport du calcium et du potassium à $40 \mathrm{mM}$ a 
provoqué une amélioration de croissance significative $(p=0,001)$ uniquement pour le $\mathrm{KNO}_{3}(97,90 \%)$. Une légère diminution non significative $(10,47 \%)$ de croissance a même été observée avec l'apport de $\mathrm{K}_{2} \mathrm{SO}_{4}$ (fig. 3-A). Avec l'apport du calcium et du potassium à $60 \mathrm{mM}$ (fig. 3-B), une tendance similaire a été observée comme pour l'apport de $40 \mathrm{mM}$ caractérisée par une amélioration significative de croissance $(p=0,001)$ uniquement pour les apports de $\mathrm{CaCl}_{2}$ et $\mathrm{KNO}_{3}$.

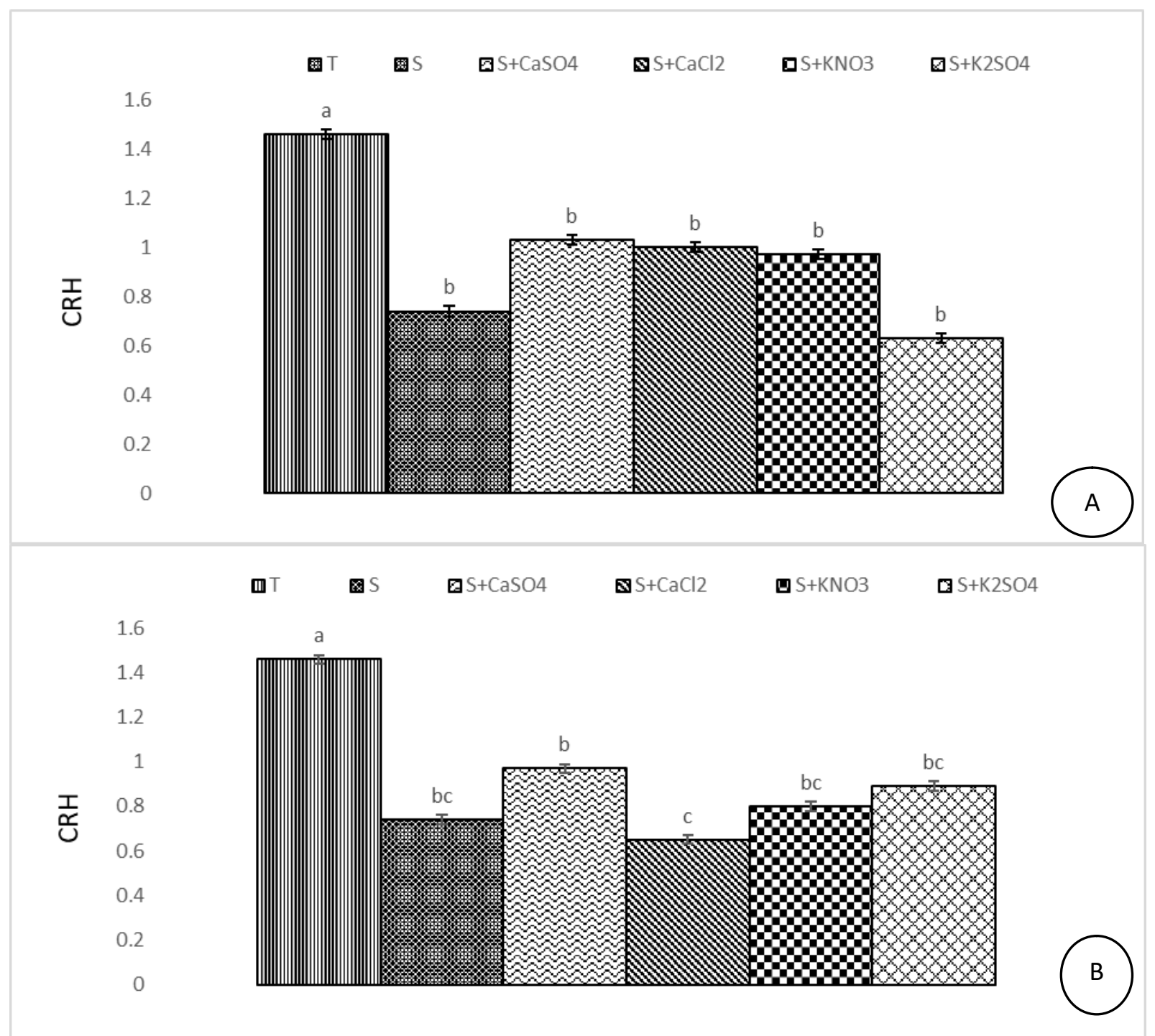

Figure 1: Effet d'un apport extérieur de calcium et de potassium sous différentes formes sur la croissance en hauteur des plants d'amarante cultivés sous stress salin après deux semaines ( $n=4$; la barre verticale correspond à l'erreur standard). (A) : Apport de $40 \mathrm{mM}$ des différents composés ; (B) : Apport de $60 \mathrm{mM}$ des différents composés Les moyennes portant des lettres différentes sont significativement différentes au seuil de 1\%. 


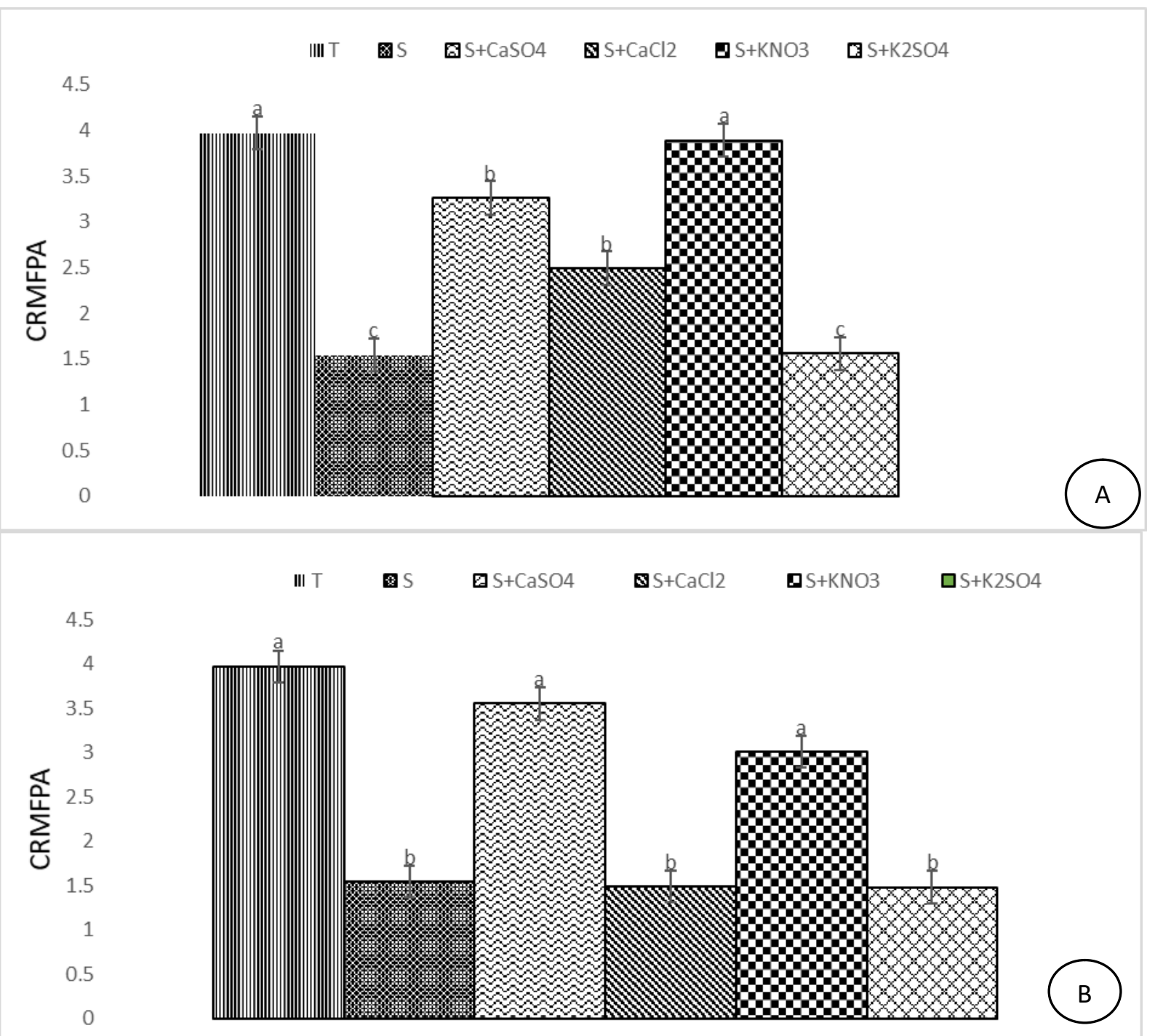

Figure 2: Effet d'un apport extérieur de calcium et de potassium sous différentes formes sur la matière fraîche de la partie aérienne des plants d'amarante cultivés sous stress salin après deux semaines $(n=4$; la barre verticale correspond à l'erreur standard). (A): Apport de $40 \mathrm{mM}$ des différents composés; (B): Apport de $60 \mathrm{mM}$ des différents composés

Les moyennes portant des lettres différentes sont significativement différentes au seuil de 1\%. 


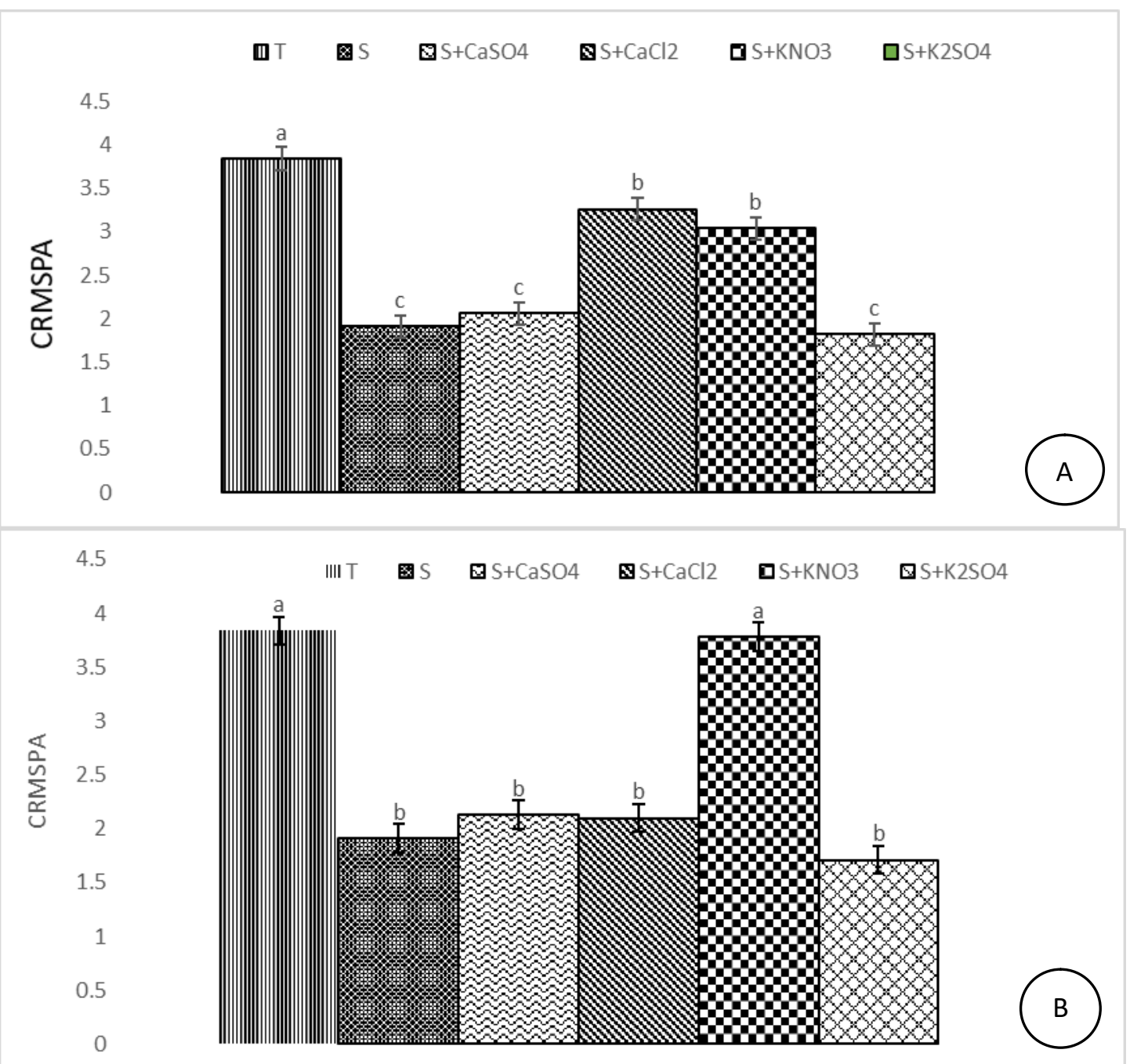

Figure 3: Effet d'un apport extérieur de calcium et de potassium sous différentes formes sur la matière sèche de la partie aérienne des plants d'amarante cultivés sous stress salin après deux semaines $(n=4$; la barre verticale correspond à l'erreur standard): (A): Apport de $40 \mathrm{mM}$ des différents composés; (B): Apport de $60 \mathrm{mM}$ des différents composés

Les moyennes portant des lettres différentes sont significativement différentes au seuil de 1\%o.

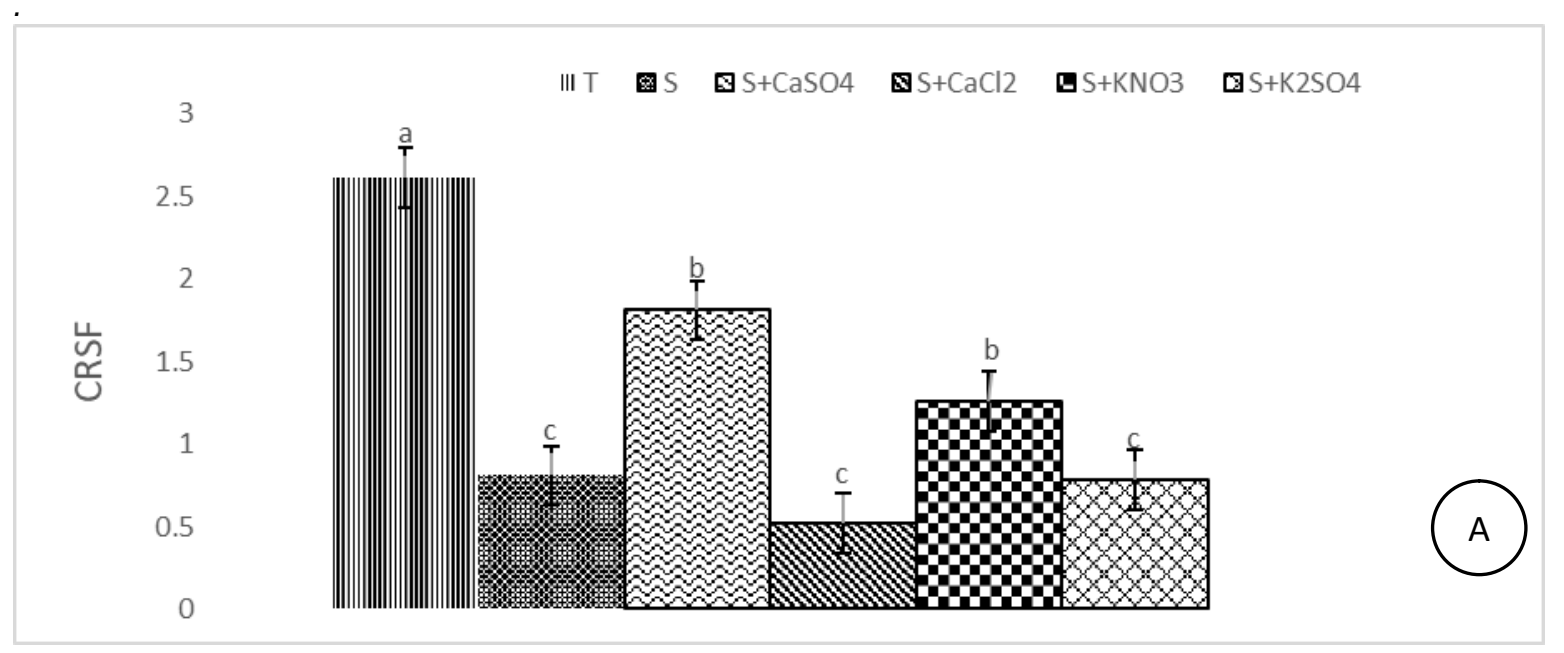




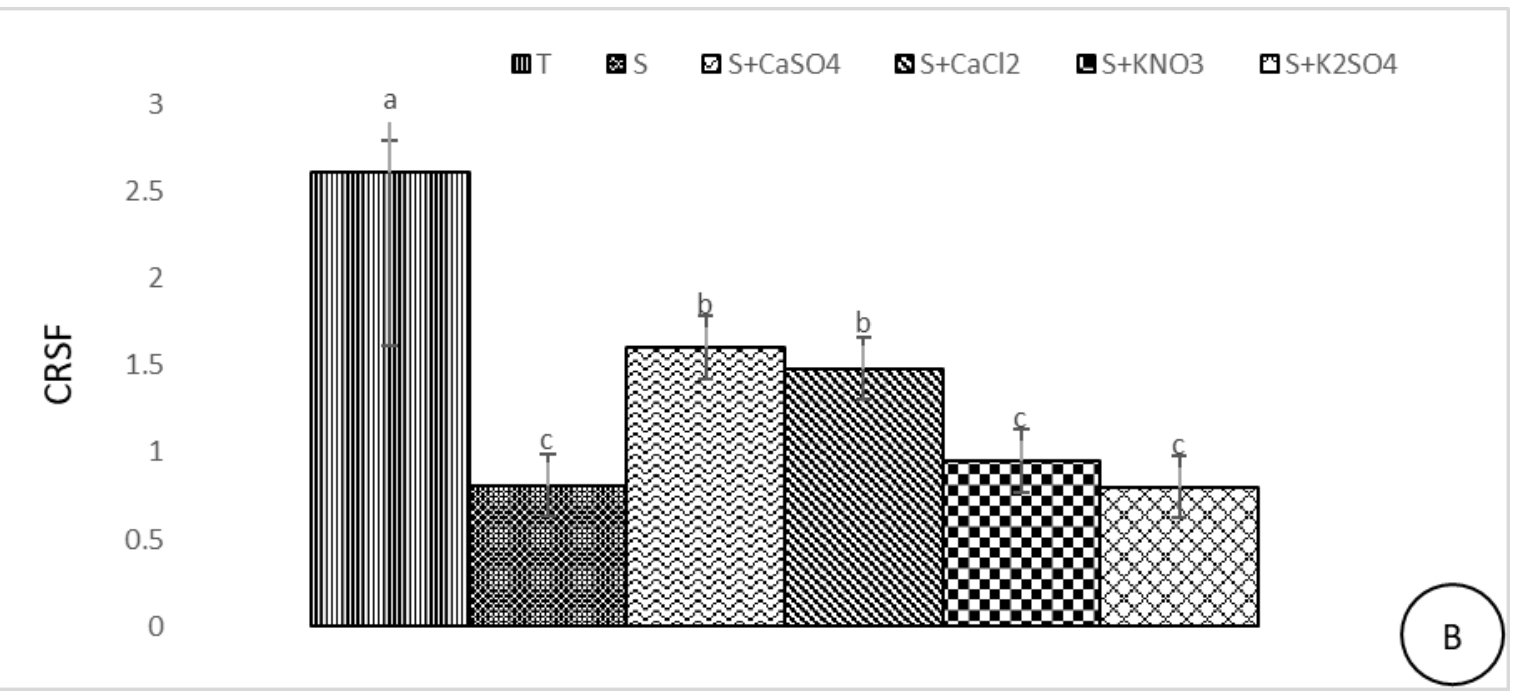

Figure 4: Effet d'un apport extérieur de calcium et de potassium sous différentes formes sur la surface foliaire des plants d'amarante cultivés sous stress salin après deux semaines $(n=4$; la barre verticale correspond à l'erreur standard) : : (A) : Apport de $40 \mathrm{mM}$ des différents composés ; (B) : Apport de $60 \mathrm{mM}$ des différents composés Les moyennes portant des lettres différentes sont significativement différentes au seuil de $1 \%$.

La salinité a provoqué une réduction significative $(p=0,001)$ de la croissance de la surface foliaire (CRSF) des plantes mais l'apport du calcium et du potassium à $40 \mathrm{mM}$ a induit une amélioration significative $(p=0,001)$ de croissance uniquement avec les apports de $\mathrm{CaSO}_{4}$ $(123,46 \%)$ et de $\mathrm{KNO}_{3}(55,55 \%)$. Une diminution non significative de croissance a même été observée avec l'apport de $\mathrm{CaCl}_{2}(35,80 \%)$ et $\mathrm{K}_{2} \mathrm{SO}_{4}(3,71 \%)$ (fig. $\left.4-\mathrm{A}\right)$. Avec l'apport du calcium et du potassium à $60 \mathrm{mM}$ (fig. 4-B), une tendance similaire a été observée comme pour l'apport de $40 \mathrm{mM}$ caractérisée par une amélioration significative de croissance $(p=0,001)$ uniquement pour les apports de $\mathrm{CaSO}_{4}$ et $\mathrm{CaCl}_{2}$.

Effet des composés apportés sur la croissance racinaire: Le tableau 1 présente l'effet d'un apport extérieur des quatre composés sur la croissance des racines des plantes d'amarante cultivées en présence de stress salin pendant deux semaines. Sous l'effet du $\mathrm{NaCl}$, la croissance des racines a été significativement diminuée $(p=0,001)$ quel que soit le paramètre de croissance considéré. L'apport des différents composés à $40 \mathrm{mM}$ a provoqué une amélioration significative $(p=$ $0,05)$ de la longueur de la racine uniquement pour le $\mathrm{CaCl}_{2}(370,58 \%)$ et le $\mathrm{KNO}_{3}(376,47 \%)$. Pour la masse fraiche des racines, l'augmentation de croissance induite par les apports de calcium et de potassium n'a été significative $(\mathrm{p}=0,05)$ que pour le $\mathrm{KNO}_{3}(38,89 \%)$ et le $\mathrm{K}_{2} \mathrm{SO}_{4}(38,89 \%)$. En ce qui concerne la masse sèche des racines, l'augmentation de croissance observée n'est significative $(p=0,001)$ que pour le $\mathrm{KNO}_{3}$ (110,28\%). L'apport de $60 \mathrm{mM}$ des composés n'a induit aucune amélioration par rapport à $40 \mathrm{mM}$. Le $\mathrm{KNO}_{3}$ a ainsi été le seul composé à induire des améliorations significatives sur tous les paramètres de croissance racinaire pris en compte. $\mathrm{Le}_{2} \mathrm{SO}_{4}$ et le $\mathrm{CaCl}_{2}$ n'ont provoqué d'amélioration significative que sur un seul des trois paramètres considérés au niveau racinaire. Dans l'ensemble, l'apport de $\mathrm{KNO}_{3}$ à $40 \mathrm{mM}$ a eu un effet améliorateur significatif sur six des sept paramètres de la croissance pris en compte dont trois (03) des quatre (04) paramètres de la partie aérienne. II a été suivi par le $\mathrm{CaSO}_{4}$ qui a eu un effet améliorateur sur tous les paramètres de la croissance de la partie aérienne pris en compte avec un effet significatif sur deux des 4 paramètres. Le $\mathrm{CaCl}_{2}$ quant à lui a eu un effet améliorateur sur trois des quatre paramètres pris en compte avec un effet significatif sur un seul des 4 paramètres. Le $\mathrm{K}_{2} \mathrm{SO}_{4}$ n'a eu aucun effet améliorateur sur la croissance de la partie aérienne des plantes sous stress salin. A $60 \mathrm{mM}$, la même tendance a été maintenue sauf que l'efficacité du $\mathrm{KNO}_{3}$ et du $\mathrm{K}_{2} \mathrm{SO}_{4}$ a diminué au niveau racinaire et celle du $\mathrm{KNO}_{3}$ a diminué au niveau de la partie aérienne; celle du $\mathrm{CaCl}_{2}$ a augmenté au niveau de la partie aérienne notamment pour la masse sèche de la partie aérienne et la surface foliaire. 
Tableau 1: Effet d'un apport extérieur de calcium et de potassium sous différentes formes sur la croissance racinaire des plants d'amarante cultivés sous stress pendant deux semaines. Les valeurs sont présentées sous forme de moyenne \pm erreur standard $(n=4)$.

\begin{tabular}{cllll} 
& & $\mathbf{L}$ & MF & MS \\
\hline & $\mathrm{T}$ & $0,97 \pm 0,12^{\mathrm{a}}$ & $2,62 \pm 0,12^{\mathrm{a}}$ & $2,36 \pm 0,20^{\mathrm{a}}$ \\
& $\mathrm{S}$ & $0,17 \pm 0,06^{\mathrm{d}}$ & $1,44 \pm 0,09^{\mathrm{c}}$ & $1,07 \pm 0,16^{\mathrm{c}}$ \\
\hline $\mathrm{CaSO}_{4}$ & $\mathrm{~S}+40$ & $0,57 \pm 0,10^{\mathrm{abcd}}$ & $1,57 \pm 0,11^{\mathrm{bc}}$ & $1,40 \pm 0,10^{\mathrm{bc}}$ \\
$\mathrm{CaCl}_{2}$ & $\mathrm{~S}+60$ & $0,38 \pm 0,04^{\mathrm{bcd}}$ & $1,51 \pm 0,08^{\mathrm{bc}}$ & $1,48 \pm 0,08^{\mathrm{bc}}$ \\
\hline & $\mathrm{S}+40$ & $0,80 \pm 0,12^{\mathrm{ab}}$ & $0,88 \pm 0,12^{\mathrm{d}}$ & $1,06 \pm 0,13^{\mathrm{c}}$ \\
$\mathrm{KNO}_{3}$ & $\mathrm{~S}+60$ & $0,66 \pm 0,14^{\mathrm{abc}}$ & $0,48 \pm 0,10^{\mathrm{d}}$ & $1,23 \pm 0,11^{\mathrm{c}}$ \\
\hline & $\mathrm{S}+40$ & $0,81 \pm 0,06^{\mathrm{ab}}$ & $2,00 \pm 0,13^{\mathrm{b}}$ & $2,25 \pm 0,083^{\mathrm{ab}}$ \\
$\mathrm{K}_{2} \mathrm{SO}_{4}$ & $\mathrm{~S}+60$ & $0,74 \pm 0,05^{\mathrm{ab}}$ & $1,48 \pm 0,16^{\mathrm{bc}}$ & $2,26 \pm 0,26^{\mathrm{ab}}$ \\
\hline & $\mathrm{S}+40$ & $0,23 \pm 0,06^{\mathrm{cd}}$ & $2,00 \pm 0,13^{\mathrm{b}}$ & $1,13 \pm 0,31^{\mathrm{c}}$ \\
\hline
\end{tabular}

Les moyennes avec des lettres différentes dans la même colonne sont significativement différentes au seuil de $1 \%$.

$L$ : Longueur ; MF : Masse fraîche ; MS : Masse sèche

Effet du $\mathrm{NaCl}$ et des composés apportés sur la teneur en ions des plantes: Le tableau 2 présente l'effet d'un apport extérieur des quatre composés sur les teneurs en sodium et en potassium des feuilles et des racines des plantes d'amarante cultivées en présence de stress salin pendant deux semaines. Sous l'effet du $\mathrm{NaCl}$, la teneur en sodium des feuilles a subi une augmentation significative $(p=0,001)$. Avec les composés apportés, cette teneur a subi une diminution de $47,51 \% ; 14,97 \% ; 24,55 \%$ et $2,40 \%$ par rapport aux plantes stressées respectivement pour les apports de $\mathrm{CaSO}_{4}, \mathrm{CaCl}_{2}, \mathrm{KNO}_{3}$ et $\mathrm{K}_{2} \mathrm{SO}_{4}$. Toutefois, les diminutions observées ne sont significatives $(p=0,05)$ que pour les apports de $\mathrm{CaSO}_{4}$; de $\mathrm{CaCl}_{2}$ et de $\mathrm{KNO}_{3}$. Ainsi, seul l'apport de $\mathrm{K}_{2} \mathrm{SO}_{4}$ n'a pas induit une diminution significative de la teneur en $\mathrm{Na}^{+}$des feuilles. Dans les racines, la teneur en sodium a subi une augmentation significative $(p=0,001)$ sous l'effet $d u$ $\mathrm{NaCl}$. Avec les composés apportés, cette teneur a connu une diminution de $32,43 \% ; 28,28 \% ; 43,61 \%$ et $27 \%$ respectivement pour les apports de $\mathrm{CaSO}_{4}, \mathrm{CaCl}_{2}$, $\mathrm{KNO}_{3}$ et $\mathrm{K}_{2} \mathrm{SO}_{4}$. Les diminutions observées sont significatives $(p=0,05)$ pour tous les composés apportés. Sous l'effet du $\mathrm{NaCl}$, la teneur en potassium des feuilles a subi une diminution significative $(p=0,001)$. Avec les composés apportés à $40 \mathrm{mM}$, cette teneur a connu une augmentation de $845,25 \%$; $141,10 \% ; 220,56 \%$ et $141,10 \%$ par rapport aux plantes stressées respectivement pour les apports de $\mathrm{CaSO}_{4}$, $\mathrm{CaCl}_{2}, \mathrm{KNO}_{3}$ et $\mathrm{K}_{2} \mathrm{SO}_{4}$. Les augmentations observées sont significatives $(p=0,05)$ uniquement pour le $\mathrm{CaSO}_{4}$ et le $\mathrm{KNO}_{3}$. Dans les racines, la teneur en potassium a subi une diminution significative $(p=0,001)$. Avec les composés apportés, cette teneur a connu une augmentation significative $(p=0,05)$ de $61,49 \%$; $114,94 \%$ et $68,96 \%$ par rapport aux plantes stressées respectivement pour les apports de $\mathrm{CaSO}_{4}, \mathrm{KNO}_{3}$ et $\mathrm{K}_{2} \mathrm{SO}_{4}$, et une diminution de $17,24 \%$ pour l'apport de $\mathrm{CaCl}_{2}$. Avec l'apport de $60 \mathrm{mM}$ de composés, seul le $\mathrm{CaCl}_{2}$ a induit une amélioration de l'absorption du potassium meilleure que l'apport de $40 \mathrm{mM}$ au niveau des feuilles. Dans l'ensemble, les apports de $\mathrm{CaSO}_{4}$ et de $\mathrm{KNO}_{3}$ ont eu les meilleurs effets améliorateurs sur la nutrition minérale des plantes au niveau de la partie aérienne (diminution de $\mathrm{Na}^{+}$et augmentation de $\mathrm{K}^{+}$par rapport aux plants stressés), mais aussi au niveau de l'alimentation en $\mathrm{Na}^{+}$des racines (diminution). $\mathrm{Au}$ niveau racinaire, les apports de $\mathrm{KNO}_{3}$ et de $\mathrm{K}_{2} \mathrm{SO}_{4}$ ont induit la meilleure alimentation en $\mathrm{K}^{+}$(augmentation). Cependant, seul l'apport de $\mathrm{K}_{2} \mathrm{SO}_{4}$ n'a pas induit une diminution significative de la teneur en $\mathrm{Na}^{+}$des feuilles. Par ailleurs, seul l'apport de $\mathrm{CaSO}_{4}$ au niveau des feuilles a donné une teneur de $\mathrm{Na}^{+}$inférieure à celle du témoin; et une teneur de $\mathrm{K}^{+}$supérieure à celle du témoin. De même, seul l'apport de $\mathrm{KNO}_{3}$ au niveau des racines a donné une teneur de $\mathrm{Na}^{+}$inférieure à celle du témoin. Sous l'effet $\mathrm{du} \mathrm{NaCl}$, le rapport $\mathrm{K} / \mathrm{Na}$ des feuilles a subi une diminution significative $(p=0,001)$. Avec les composés apportés à $40 \mathrm{mM}$, ce rapport a connu une augmentation significative $(p=0,001)$ de $1692,36 \% ; \quad 183,71 \% ; \quad 324,93 \%$ et $147,82 \%$ respectivement pour les apports de $\mathrm{CaSO}_{4}, \mathrm{CaCl}_{2}$, $\mathrm{KNO}_{3}$ et $\mathrm{K}_{2} \mathrm{SO}_{4}$. Dans les racines, sous l'effet du $\mathrm{NaCl}$, le rapport $\mathrm{K} / \mathrm{Na}$ des feuilles a subi une diminution significative $(p=0,001)$. Avec les composés apportés, ce rapport a connu une augmentation de 138,83 ; $15,19 \% ; 280,38 \%$ et $131,02 \%$ par rapport aux plantes stressées respectivement pour les apports de $\mathrm{CaSO}_{4}$, 
$\mathrm{CaCl}_{2}, \mathrm{KNO}_{3}$ et $\mathrm{K}_{2} \mathrm{SO}_{4}$. Cependant, l'augmentation observée n'est significative que pour les apports de $\mathrm{CaSO}_{4}, \mathrm{KNO}_{3}$ et $\mathrm{K}_{2} \mathrm{SO}_{4}$. Avec l'apport de $60 \mathrm{mM}$ de composés, seul le $\mathrm{CaCl}_{2}$ a induit une amélioration du rapport $\mathrm{K} / \mathrm{Na}$ meilleure que l'apport de $40 \mathrm{mM}$ et significative au niveau des feuilles.

Tableau 2 : Effet d'un apport extérieur de potassium et de calcium sous différentes formes sur la teneur en ions sodium et potassium des feuilles et des racines de plants d'amarante cultivés sous stress salin. Les valeurs sont présentées sous forme de moyenne \pm erreur standard $(n=4)$.

\begin{tabular}{clllll}
\hline & & \multicolumn{2}{c}{ Feuilles } & \multicolumn{2}{c}{ Racines } \\
\cline { 3 - 6 } & & \multicolumn{1}{c}{$\mathrm{Na}$} & \multicolumn{1}{c}{$\mathrm{K}$} & $\mathrm{Na}$ & \multicolumn{1}{c}{$\mathrm{K}$} \\
\hline & $\mathrm{T}$ & $3,24 \pm 0,07 \mathrm{e}$ & $337,38 \pm 9,73 \mathrm{~b}$ & $4,20 \pm 0,07 \mathrm{c}$ & $1201,08 \pm 22,09 \mathrm{a}$ \\
& $\mathrm{S}$ & $5,01 \pm 0,02 \mathrm{a}$ & $98,51 \pm 1,34 \mathrm{c}$ & $6,26 \pm 0,10 \mathrm{a}$ & $469,64 \pm 9,35 \mathrm{~d}$ \\
\hline $\mathrm{CaSO}_{4}$ & $\mathrm{~S}+40$ & $2,63 \pm 0,05 \mathrm{f}$ & $931,17 \pm 93,38 \mathrm{a}$ & $4,23 \pm 0,07 \mathrm{c}$ & $758,43 \pm 15,02 \mathrm{c}$ \\
& $\mathrm{S}+60$ & $2,60 \pm 0,13 \mathrm{f}$ & $831,30 \pm 26,58 \mathrm{a}$ & $4,20 \pm 0,07 \mathrm{c}$ & $836,71 \pm 31,12 \mathrm{c}$ \\
\hline & $\mathrm{S}+40$ & $4,26 \pm 0,10 \mathrm{~b}$ & $237,51 \pm 7,14 \mathrm{bc}$ & $4,49 \pm 0,10 \mathrm{bc}$ & $388,66 \pm 14,02 \mathrm{~d}$ \\
$\mathrm{CaCl}_{2}$ & $\mathrm{~S}+60$ & $4,00 \pm 0,08 \mathrm{bc}$ & $306,34 \pm 11,04 \mathrm{~b}$ & $4,86 \pm 0,07 \mathrm{~b}$ & $391,36 \pm 7,14 \mathrm{~d}$ \\
\hline & $\mathrm{S}+40$ & $3,78 \pm 0,02 \mathrm{~cd}$ & $315,78 \pm 4,67 \mathrm{~b}$ & $3,53 \pm 0,00 \mathrm{~d}$ & $1009,45 \pm 37,78 \mathrm{~b}$ \\
$\mathrm{KNO}_{3}$ & $\mathrm{~S}+60$ & $3,59 \pm 0,07 \mathrm{de}$ & $326,58 \pm 4,67 \mathrm{~b}$ & $3,47 \pm 0,13 \mathrm{~d}$ & $1063,43 \pm 33,16 \mathrm{~b}$ \\
\hline & $\mathrm{S}+40$ & $4,89 \pm 0,12 \mathrm{a}$ & $237,51 \pm 7,14 \mathrm{bc}$ & $4,57 \pm 0,12 \mathrm{bc}$ & $793,52 \pm 4,67 \mathrm{c}$ \\
$\mathrm{K}_{2} \mathrm{SO}_{4}$ & $\mathrm{~S}+60$ & $4,95 \pm 0,05 \mathrm{a}$ & $202,42 \pm 4,67 \mathrm{bc}$ & $6,08 \pm 0,05 \mathrm{a}$ & $777,33 \pm 4,67 \mathrm{c}$ \\
\hline
\end{tabular}

Les moyennes avec des lettres différentes dans la même colonne sont significativement différentes au seuil de $5 \%$.

$T=0 \mathrm{mM} \mathrm{NaCl} ; \mathrm{S}=120 \mathrm{mM} \mathrm{NaCl} ; \mathrm{S}+40 \mathrm{mM}$ et $\mathrm{S}+60 \mathrm{mM}$ de $\mathrm{CaCl}_{2}$, de $\mathrm{CaSO}_{4}$, de $\mathrm{KNO}_{3}$ et de $\mathrm{K}_{2} \mathrm{SO}_{4}$

Tableau 3 : Effet d'un apport extérieur de calcium et de potassium sous différentes formes sur le rapport K/Na dans les feuilles et dans les racines des plants d'amarante cultivés sous stress salin.

\begin{tabular}{clll}
\hline & & Feuilles & Racines \\
\hline & $\mathrm{T}$ & $104,09 \pm 4,50^{\mathrm{b}}$ & $285,79 \pm 2,60^{\mathrm{a}}$ \\
& $\mathrm{S}$ & $19,65 \pm 0,38^{\mathrm{e}}$ & $75,09 \pm 2,69^{\mathrm{d}}$ \\
\hline & $\mathrm{S}+40$ & $352,20 \pm 30,00^{\mathrm{a}}$ & $179,34 \pm 4,96^{\mathrm{b}}$ \\
$\mathrm{CaSO}_{4}$ & $\mathrm{~S}+60$ & $319,26 \pm 5,86^{\mathrm{a}}$ & $199,42 \pm 10,61^{\mathrm{b}}$ \\
\hline & $\mathrm{S}+40$ & $55,75 \pm 1,29^{\mathrm{bcde}}$ & $86,50 \pm 2,20^{\mathrm{d}}$ \\
$\mathrm{CaCl}_{2}$ & $\mathrm{~S}+60$ & $76,73 \pm 4,03^{\mathrm{bcd}}$ & $80,41 \pm 2,29^{\mathrm{d}}$ \\
\hline & $\mathrm{S}+40$ & $83,50 \pm 1,59^{\mathrm{bcd}}$ & $285,63 \pm 11,06^{\mathrm{a}}$ \\
$\mathrm{KNO}_{3}$ & $\mathrm{~S}+60$ & $90,95 \pm 2,16^{\mathrm{bc}}$ & $305,90 \pm 2,71^{\mathrm{a}}$ \\
\hline & $\mathrm{S}+40$ & $48,55 \pm 2,18^{\text {cde }}$ & $173,47 \pm 3,81^{\mathrm{b}}$ \\
$\mathrm{K}_{2} \mathrm{SO}_{4}$ & $\mathrm{~S}+60$ & $40,84 \pm 0,81^{\text {de }}$ & $127,70 \pm 0,28^{\mathrm{c}}$ \\
\hline & & Feuilles & Racines \\
\hline & $\mathrm{S}$ & $104,09 \pm 4,50^{\mathrm{b}}$ & $285,79 \pm 2,60^{\mathrm{a}}$ \\
& $\mathrm{S}+40$ & $19,65 \pm 0,38^{\mathrm{e}}$ & $75,09 \pm 2,69^{\mathrm{d}}$ \\
\hline & $\mathrm{S}+60$ & $352,20 \pm 30,00^{\mathrm{a}}$ & $179,34 \pm 4,96^{\mathrm{b}}$ \\
$\mathrm{CaSO}_{4}$ & $\mathrm{~S}+40$ & $319,26 \pm 5,86^{\mathrm{a}}$ & $199,42 \pm 10,61^{\mathrm{b}}$ \\
\hline & $\mathrm{S}+60$ & $55,75 \pm 1,29^{\mathrm{bcde}}$ & $86,50 \pm 2,20^{\mathrm{d}}$ \\
$\mathrm{CaCl}_{2}$ & $\mathrm{~S}+40$ & $76,73 \pm 4,03^{\mathrm{bcd}}$ & $80,41 \pm 2,29^{\mathrm{d}}$ \\
\hline & $\mathrm{S}+60$ & $83,50 \pm 1,59^{\mathrm{bcd}}$ & $285,63 \pm 11,06^{\mathrm{a}}$ \\
$\mathrm{KNO}_{3}$ & $\mathrm{~S}+40$ & $90,95 \pm 2,16^{\mathrm{bc}}$ & $305,90 \pm 2,71^{\mathrm{a}}$ \\
\hline $\mathrm{K}_{2} \mathrm{SO}_{4}$ & $\mathrm{~S}+60$ & $48,55 \pm 2,18^{\text {cde }}$ & $173,47 \pm 3,81^{\mathrm{b}}$ \\
\hline
\end{tabular}

Les moyennes avec des lettres différentes dans la même colonne sont significativement différentes au seuil de 1\%.

$T=0 \mathrm{mM} \mathrm{NaCl} ; \mathrm{S}=120 \mathrm{mM} \mathrm{NaCl} ; \mathrm{S}+40 \mathrm{mM}$ et $\mathrm{S}+60 \mathrm{mM}$ de $\mathrm{CaCl}_{2}$, de $\mathrm{CaSO}_{4}$, de $\mathrm{KNO}_{3}$ et de $\mathrm{K}_{2} \mathrm{SO}_{4}$ 
Dans l'ensemble, tous les apports ont amélioré de façon notable le rapport $\mathrm{K} / \mathrm{Na}$ des feuilles avec les meilleures améliorations pour les apports de $\mathrm{CaSO}_{4}$ et de $\mathrm{KNO}_{3}$. Au niveau racinaire, seul l'apport de $\mathrm{CaCl}_{2}$ n'a pas eu un effet améliorateur significatif sur le

\section{DISCUSSION}

Effet de la salinité sur la croissance des plantes : Le stress salin a provoqué une inhibition évidente de la croissance de tous les paramètres de la partie aérienne et de la partie racinaire des plantes d'amarante. II s'agit d'une tendance générale chez toutes les plantes cultivées comme rapporté chez de nombreuses espèces incluant le riz (Prodjinoto et al., 2018), la canne à sucre (Gandonou et al., 2012 ; Gandonou et Skali-Senhaji, 2015), la tomate (Ould Mohamdi et al., 2011) et le piment (R'him et al., 2013 ; Kpinkoun et al., 2018). Chez l'amarante, plusieurs auteurs ont signalé que le stress salin a réduit la croissance de différents génotypes (Qin et al., 2013 ; Ratnakar et Rai, 2013; Amukali et al., 2015; Lavini et al., 2016 ; Wouyou et al., 2017 ; Gandonou et al., 2018). Les réductions de croissance observées dans notre étude sont significatives pour tous les paramètres de croissance. Selon Odjegba et Chukwunwike (2012), la réduction de la croissance des plantes sous stress salin, en particulier dans l'accumulation de la biomasse, pourrait être la conséquence d'un stress hydrique résultant d'une diminution du potentiel osmotique externe et/ou de l'accumulation d'ions devenant toxiques.

Effet des différents composés apportés sur la croissance des plantes: Au niveau de la partie aérienne, aussi bien à $40 \mathrm{mM}$ qu'à $60 \mathrm{mM}$, le $\mathrm{KNO}_{3}$ a été le plus efficace quant à sa capacité à atténuer les effets néfastes du $\mathrm{NaCl}$ sur la croissance des plantes, suivi par le $\mathrm{CaSO}_{4}$ puis le $\mathrm{CaCl}_{2}$. Le $\mathrm{K}_{2} \mathrm{SO}_{4}$ quant à lui n'a eu aucun effet améliorateur sur la croissance de la partie aérienne des plantes. A $60 \mathrm{mM}$, seul l'efficacité du $\mathrm{CaCl}_{2}$ a été améliorée ; celle des autres composés a même baissé à travers la diminution du nombre de paramètres dont la croissance a été significativement améliorée. Au niveau racinaire, les apports de $40 \mathrm{mM}$ de $\mathrm{KNO}_{3}$ a été le plus efficace dans sa capacité à atténuer les effets négatifs du $\mathrm{NaCl}$ sur la croissance, suivi par le $\mathrm{CaCl}_{2}$ et le $\mathrm{K}_{2} \mathrm{SO}_{4}$. De plus aucune amélioration de croissance n'a été observée pour aucun des composés avec l'apport de $60 \mathrm{mM}$ au niveau racinaire en termes de nombre de paramètres. Ainsi, le $\mathrm{CaSO}_{4}$ qui a été l'un des composés les plus efficaces pour l'atténuation des effets négatifs de la salinité au niveau de la partie aérienne apparaît comme le plus rapport K/Na. Cependant, seul l'apport de $\mathrm{CaSO}_{4}$ au niveau des feuilles et de $\mathrm{KNO}_{3}$ au niveau des racines ont donné des valeurs de $\mathrm{K} / \mathrm{Na}$ supérieures ou égales à celle du témoin.

inefficace au niveau racinaire. Ce résultat indique que l'effet améliorateur des différents composés dépend à la fois de l'organe pris en compte mais aussi de la dose considérée. Cependant, le $\mathrm{K}_{2} \mathrm{SO}_{4}$ qui n'a eu aucun effet améliorateur sur la croissance des plantes au niveau de la partie aérienne, n'a également eu que trop peu d'effet améliorateur sur la croissance de la partie racinaire. Ce résultat indique que ce composé n'est pas adapté pour atténuer les effets négatifs de la salinité sur les plantes d'amarante. De même, le $\mathrm{KNO}_{3}$ a confirmé sa grande capacité à atténuer les effets du $\mathrm{NaCl}$ sur la croissance de l'amarante quel que soit l'organe pris en compte et quelle que soit la dose utilisée avec la meilleure efficacité pour la dose de 40 mM. II apparaît de ce fait comme le meilleur candidat à l'atténuation des effets de la salinité chez l'amarante, suivi par le CaSO4. Chez d'autres génotypes d'amarante, Omami et Hammes (2006) montré que le calcium apporté sous forme de $\mathrm{CaSO}_{4}$ avait réduit les effets négatifs induits par la concentration élevée de $\mathrm{NaCl}$ sur la croissance des plants; mais cette réduction a été plus importante avec l'apport de calcium sous forme de $\mathrm{CaSO}_{4}$ comparativement au $\mathrm{CaCl}_{2}$. Ces auteurs ont justifié cette différence par l'apport supplémentaire de $\mathrm{Cl}^{-}$par le $\mathrm{CaCl}_{2}$ qui est lui-même toxique pour les plantes. Ces données corroborent nos résultats et suggèrent que la faible efficacité $\mathrm{du} \mathrm{CaCl}_{2}$ à atténuer les effets dépressifs du $\mathrm{NaCl}$ sur la croissance des plantes en comparaison avec le $\mathrm{CaSO}_{4}$ serait due à la toxicité liée à l'apport supplémentaire de $\mathrm{Cl}$ Cependant, l'effet améliorateur du $\mathrm{CaCl}_{2}$ a été meilleur avec une dose de $60 \mathrm{mM}$ donc supérieure à $40 \mathrm{mM}$. Ceci semble indiquer que la toxicité du $\mathrm{Cl}^{-}$seul ne suffit pas expliquer la faible efficacité de ce composé à 40 $\mathrm{mM}$. Cette amélioration de croissance à $60 \mathrm{mM}$ a été associée également à une amélioration de l'absorption du potassium dans les feuilles sans un changement au niveau de l'absorption du sodium. On pourrait donc supposer qu'une augmentation des teneurs en ions $\mathrm{Ca}^{++}$au-dessus d'un certain seuil en présence de 60 $\mathrm{mM}$ de $\mathrm{CaCl}_{2}$ aurait favorisé l'absorption du potassium, ce qui expliquerait l'amélioration de l'efficacité du $\mathrm{CaCl}_{2}$ observée. Des effets améliorateurs d'un apport extérieur de calcium ont été également rapportés chez 
d'autres plantes (Kaya et al., 2002 ; Ebert et al., 2002) exposés au stress salin. Cependant, il a été rapporté chez le tournesol que l'apport supplémentaire de calcium n'a pas été capable d'améliorer les dommages causés par le $\mathrm{NaCl}$ (Sohan et al., 1999). Ce résultat indique que l'effet améliorateur du calcium sur les dommages causés par le $\mathrm{NaCl}$ dépend non seulement de la forme sous laquelle il est apporté mais aussi de l'espèce végétale concernée. L'effet améliorateur d'un apport extérieur de potassium sur les effets négatifs induits par la salinité a été rapporté par plusieurs auteurs (Hussain et al., 2013; Manaa et al., 2014; Chakraborty et al., 2016). Notre étude révèle que c'est seulement sous forme de $\mathrm{KNO}_{3}$ que le potassium améliore la croissance de la partie aérienne des plantes. Ceci indique que l'effet améliorateur du potassium sur la croissance de la partie aérienne des plantes dépend de la forme sous laquelle il est apporté comme c'est le cas pour le calcium. Une augmentation de la dose des composés a induit une réduction de l'efficacité pour le $\mathrm{KNO}_{3}$, le $\mathrm{CaSO}_{4}$ et le $\mathrm{K}_{2} \mathrm{SO}_{4}$ indiquant que cette dose de $60 \mathrm{mM}$ est excessive pour ces trois composés. Dans l'ensemble, le $\mathrm{KNO}_{3}$ apparaît comme le meilleur candidat à l'atténuation des effets de la salinité chez l'amarante, suivi par le $\mathrm{CaSO}_{4}$.

Relation entre l'amélioration de la croissance par les composés apportés et la nutrition minérale de la plante : L'effet améliorateur d'un apport extérieur de calcium ou de potassium sur les dommages induits par la salinité a généralement été associé au maintien d'un rapport $\mathrm{K}^{+} / \mathrm{Na}^{+}$optimal et à une homéostasie dans le cytosol en relation avec une inhibition de l'influx de $\mathrm{Na}^{+}$, de l'efflux de $\mathrm{K}^{+}$ou la promotion de l'efflux de $\mathrm{Na}^{+}$ (sortie de la cellule) et l'influx de $\mathrm{K}^{+}$(entrée dans la cellule) à travers la membrane plasmique (Elphick et al., 2001; Demidchik et Tester, 2002; Shabala et al., 2006 ; Hussain et al., 2013; Chakraborty et al., 2016). Nos résultats ont révélé que les apports de $\mathrm{CaSO}_{4}$ et de $\mathrm{KNO}_{3}$ ont eu les meilleurs effets améliorateurs sur la nutrition minérale des plants au niveau de la partie aérienne (diminution de $\mathrm{Na}^{+}$et augmentation de $\mathrm{K}^{+}$par rapport aux plants stressés), mais aussi au niveau de l'alimentation en $\mathrm{Na}^{+}$des racines (diminution par rapport aux plants stressés). Au niveau racinaire, les apports de $\mathrm{KNO}_{3}$ et de $\mathrm{K}_{2} \mathrm{SO}_{4}$ ont induit la meilleure alimentation en $\mathrm{K}^{+}$(augmentation par rapport aux plants stressés). Ces résultats indiquent que l'efficacité du $\mathrm{KNO}_{3}$ et du $\mathrm{CaSO}_{4}$ à atténuer les effets négatifs de la salinité notamment au niveau de la partie aérienne est associée soit à une inhibition de l'influx $\mathrm{de}^{\mathrm{Na}^{+}}$, de l'efflux de $\mathrm{K}^{+}$ou la promotion de l'efflux de $\mathrm{Na}^{+}$(sortie de la cellule) et l'influx de $\mathrm{K}^{+}$(entrée dans la cellule) à travers la membrane plasmique. Le $\mathrm{CaCl}_{2}$ qui n'a eu qu'un effet améliorateur modéré sur la croissance des plantes notamment au niveau de la partie aérienne n'a amélioré que la teneur en sodium des feuilles à $40 \mathrm{mM}$ indiquant que l'amélioration de ce paramètre seul ne peut atténuer efficacement les effets négatifs de la salinité. Cette idée est confirmée par le fait que l'apport de $60 \mathrm{mM}$ de $\mathrm{CaCl}_{2}$ qui a provoqué une amélioration importante de la croissance a également amélioré de manière significative aussi bien la teneur en sodium des feuilles que leur teneur en potassium et le rapport $\mathrm{K} / \mathrm{Na}$. Ainsi, la combinaison entre la réduction de la teneur en sodium et l'augmentation de la teneur en potassium est nécessaire pour avoir un meilleur effet améliorateur sur la croissance des plantes d'amarante. Cependant, nos résultats ont également montré que seul l'apport de $\mathrm{CaSO}_{4}$ au niveau des feuilles a donné une teneur de $\mathrm{Na}^{+}$inférieure à celle du témoin ; et une teneur de $\mathrm{K}^{+}$supérieure à celle du témoin. Ces résultats semblent indiquer que l'efficacité du $\mathrm{CaSO}_{4}$ à atténuer les effets négatifs de la salinité au niveau de la partie aérienne est associée principalement à la promotion de l'efflux de $\mathrm{Na}^{+}$(sortie de la cellule) et l'influx de $\mathrm{K}^{+}$(entrée dans la cellule) à travers la membrane plasmique. De même, l'efficacité du $\mathrm{KNO}_{3}$ à atténuer les effets négatifs de la salinité au niveau de la partie racinaire est associée à la promotion de l'efflux de $\mathrm{Na}^{+}$(sortie de la cellule) à travers la membrane plasmique puisqu'il a induit une teneur de $\mathrm{Na}^{+}$ inférieure à celle du témoin au niveau racinaire. Nos résultats montrent l'évidence que le seul composé dont l'apport extérieur n'a pas eu d'effet améliorateur sur la croissance de la partie aérienne des plantes est aussi le seul à ne pas induire une diminution significative de la teneur en $\mathrm{Na}^{+}$des feuilles, en l'occurrence le $\mathrm{K}_{2} \mathrm{SO}_{4}$. II semble donc logique de dire que le premier mode d'action des composés apportés sur les plantes est la réduction de la teneur en $\mathrm{Na}^{+}$des feuilles indiquant que la toxicité du sodium (en l'absence du dosage du $\mathrm{Cl}$-) est le principal facteur d'inhibition de la croissance par le $\mathrm{NaCl}$ chez le cultivar d'amarante utilisé dans cette étude. Cette tendance ne semble pas confirmée au niveau racinaire puisque le $\mathrm{CaSO}_{4}$ bien que n'ayant pas provoqué d'amélioration significative de la croissance racinaire a eu tout de même un effet améliorateur significatif sur la teneur en sodium, la teneur en potassium et le rapport $\mathrm{K} / \mathrm{Na}$ des racines. Avec l'apport de $60 \mathrm{mM}$, une tendance similaire a été observée pour l'ensemble des composés à l'exception $\mathrm{du} \mathrm{CaCl}_{2}$ qui a induit une accumulation plus importante 
(une plus faible diminution) de $\mathrm{K}^{+}$et un meilleur rapport $\mathrm{K} / \mathrm{Na}$ dans les feuilles comparativement à un apport de $40 \mathrm{mM}$ bien corrélés avec l'amélioration de la croissance de la partie aérienne. $\mathrm{Le}_{2} \mathrm{SO}_{4}$ quant à lui a

\section{CONCLUSION}

Les résultats de cette étude ont révélé que la salinité a induit une réduction significative de la croissance des plants d'amarante après deux semaines d'exposition et que l'effet améliorateur du potassium et du calcium sur la croissance des plants dépend non seulement de la forme sous laquelle ils sont apportés et de la dose apportée mais aussi des organes de la plante pris en compte. $\mathrm{Le} \mathrm{KNO}_{3}$ et le $\mathrm{CaSO}_{4}$ présentent leur meilleure induit une diminution plus importante de la teneur en $\mathrm{K}^{+}$ des feuilles par rapport à l'apport de $40 \mathrm{mM}$, ce qui explique une inefficacité plus accrue.

efficacité à $40 \mathrm{mM}$ tandis que le $\mathrm{CaCl}_{2}$ présente sa meilleure efficacité à $60 \mathrm{mM}$. Le $\mathrm{KNO}_{3}$ apparaît comme le meilleur candidat à l'atténuation des effets de la salinité chez l'amarante, suivi par le $\mathrm{CaSO}_{4}$. Cet effet améliorateur est dû principalement à une meilleure exclusion du $\mathrm{Na}^{+}$des feuilles associée à une meilleure accumulation de $\mathrm{K}^{+}$et au maintien d'un meilleur rapport $\mathrm{K} / \mathrm{Na}$.

\section{REMERCIEMENTS}

Les auteurs remercient le Projet d'Appui au Développement du Maraîchage au Bénin (PADMAR/ProCaR) pour le financement partiel des travaux.

\section{REFERENCES BIBLIOGRAPHIQUES}

Adorgloh-Hessou R, 2006. Guide pour le développement de l'entreprise de production et de commercialisation de légumes de qualité dans les régions urbaines et périurbaines du Sud-Bénin. Rapport de consultation, IITA - Bénin, 86p.

Allemann J, Van Den Heever E, Viljoen J, 1996. Evaluation of Amaranthus as a possible vegetable crop. Appl. Plant Sci. 10: 1-4.

Amukali O, Obadoni BO, Mensah JK, 2015. Effects of different $\mathrm{NaCl}$ concentrations on germination and seedlings growth of Amaranthus hybridus and Celosia argentea. Afr. J. Env. Sci. Technol. 9(4):301-306.

Bhattacharjee S, 2008. Triadimef on pretreatment protects newly assembled membrane system and causes up-regulation of stress proteins in salinity stressed Amaranthus lividus L. during early germination. Journal of Environmental Biology 29 (5): 805- 810.

Chakraborty K, Bhaduri D, Meena HN, Kalariya K, 2016. External potassium (Kp) application improves salinity tolerance by promoting $\mathrm{Na}^{+}$exclusion, $\mathrm{K}^{+}-$accumulation and osmotic adjustment in contrating peanut cultivars. Plant Physiology and Biochemistry 103:143-153. DOI: 10.1016/j.plaphy.2016.02.039

Cunningham AB, Dejager PJ, Hansen LCB, 1992. The indigenous plant use programme. Foundation for Research Development, Pretoria, ISBN 1874916-03-9.

Demidchik V, Tester M, 2002. Sodium fluxes through nonselective cation channels in the plasma membrane of protoplasts from Arabodopsis root. Plant physiology 128: 379-387.

Ebert G, Eberle J, Ali-Dinar H, Ludders P, 2002. Ameliorating effects of $\mathrm{Ca}(\mathrm{NO} 3)_{2}$ on growth, mineral uptake and photosynthesis of $\mathrm{NaCl}$ stressed guava seedlings (Psidium guajava L.). Sci. Hort. 93: 125-135.

Elphick CH, Sanders D, Maathuis FJM, 2001. Critical role of divalent cations and $\mathrm{Na}^{+}$efflux in Arabidopsis thaliana salt tolerance. Plant, Cell and Environment 24:733-740.

Gandonou CB, Skali Senhaji N, 2015. Sugarcane (Saccharum sp.) salt tolerance at various developmental levels. In: Chakraborty U, Chakraborty B, Editors. Abiotic Stresses in Crop Plants, CABI Publishing, United Kingdom, ISBN-13: 978-1-78064-373-1.

Gandonou CB, Abou Y, 2018. Evaluation de la qualité de l'eau, de la dynamique saline de référence et des effets visibles de la salinité sur les cultures sensibles dans la zone d'intervention $\mathrm{du}$ projet: Saison pluvieuse, Rapport d'activité, Projet PADMAR-Bénin. ; 35.

Gandonou CB, Gnancadja SL, Abrini J, Skali Senhaji N, 2012. Salinity tolerance of some sugarcane (Saccharum sp.) cultivars in hydroponic medium. International Sugar Journal 114 (1359): 190-196. 
International Journal of Plant and Soil Science 14(3): 111. DOI: $10.9734 /$ IJPSS/2017/31611.

Gandonou CB, Prodjinoto H, Zanklan SA, Wouyou AD, Lutts S, Montcho DH, Assogba Komlan F, Mensah ACG. 2018. Effects of salinity stress on growth in relation to gas exchanges parameters and water status in amaranth (Amaranthus cruentus). International Journal of Plant Physiology and Biochemistry 10(3): 19-27.

Jiang Y, Huang B, 2001. Effet of calcium on antioxidant activities and water relations associated with heat tolerance in two cool seasons grasses. Journal of Experimental Botany 52: 341-349.

Hussain Z, Khattak RA, Irshad M, Eneji AE, 2013. Ameliorative effect of potassium sulphate on the growth and chemical composition of wheat (Triticum aestivum L.) in salt-affected soils, J. Soil Sci. Plant Nutr. 13 (2):401-415. DOI: http://dx.doi.org/10.4067/S071895162013005000032.

JMP Pro SAS Institute, 2009. JMP® 8. User Guide, Second Edition. Cary, NC: SAS Institute Inc. Cary, NC, USA.

Kaya C, Kirnak H, Higgs D, Saltali K, 2002. Supplementary calcium enhances plant growth and fruit yield in strawberry cultivars grown at high ( $\mathrm{NaCl}$ ) salinity. Sci. Hort. 93: 65-74.

Khan MN, Siddiqui M, Mohammad F, Naeem M, Khan MM, 2010. Calcium chloride and gibberellic acid protect linseed (Linum usitatissimum L.) from $\mathrm{NaCl}$ stress by in-ducing antioxidative defence system and osmoprotectant accumulation. Acta Physiologiae Plantarum 32: 121-132.

Lavini A, Pulvento C, d'Andria R, Riccardi M, 2016. Effects of saline irrigation on yield and qualitative characterization of seed of an amaranth accession grown under Mediterranean conditions. J. Agric. Sci. 154(5):858-869.

Makus DJ, 2003. Salinity and nitrogen level affect agronomic performance, leaf color and leaf mineral nutrients of vegetable amaranth. Subtropical Plant Sci. 55: 1-6.

Manaa A, Gharbi E, Mimouni H, Wasti S., Aschi-Smiti A, Lutts S, Ben A, 2014. Simultaneous application of salicylic acid and calcium improves salt tolerance in two contrasting tomato (Solanum lycopersicum) cultivars. South African Journal of Botany 95: 32-39.
Munns R, Tester M, 2008. Mechanism of salinity tolerance. Annual Review of Plant Biology 59: 651-681. 10.1146/annurev.arplant.59.032607.092911

Negrão S, Schmöckel SM, Tester M, 2017. Evaluating physiological responses of plants to salinity stress. Annals of Botany 119: 1-11.

Odjegba VJ, Chukwunwike IC, 2012. Physiological responses of Amaranthus hybridus $L$. under salinity stress. Indian Journal of Innovations and Developments 1 (10): 742-748.

Omami EN, 2005. Response of Amaranth to salinity stress, Ph. D Thesis, University of Pretoria, South Africa. $235 \mathrm{p}$.

Omami EN, Hammes PS, 2006. Ameliorative effects of calcium on growth and mineral uptake of saltstressed amaranth, South African Journal of Plant and Soil 23(3): 197-202.

DOI: 10.1080/02571862.2006.10634754

Ould Mohamdi M, Bouya D, Ould Mohamed Salem A, 2011. Etude de l'effet du stress salin $(\mathrm{NaCl})$ chez deux variétés de tomate (Campbell 33 et Mongal). International Journal of Biological and Chemical Sciences 5(3): 860-900.

Qin L, Guo S, Ai W, Tang Y, Cheng Q, Chen G, 2013. Effect of salt stress on growth and physiology in amaranth and lettuce: Implications for bioregenerative life support system. Adv. Space Res. 51:476- 482.

R'him T, Tlili I, Hnan I, llahy R, Benali A, Jebari $H$, 2013. Effet du stress salin sur le comportement physiologique et métabolique de trois variétés de piment (Capsicum annuum L.). Journal of Applied BioSciences 66: 5060 5069.

Ratnakar A, Rai A, 2013. Effect of $\mathrm{NaCl}$ salinity on $\beta$ carotene, thiamine, riboflavin and ascorbic acid contents in the leaves of Amaranthus polygamous L. var. Pusa Kirti. Octa Journal of Environmental Research 1(3):211-216.

Shalaba S, Demidchik V, Shalaba L, Cuin TA, Smith SJ, Miller AJ, Davies JM, Newman IA, 2006. Extracellular $\mathrm{Ca}^{2+}$ ameliorates $\mathrm{NaCl}$ - induced $\mathrm{K}^{+}$loss from Arabidopsis root and leaf cells by controlling plasma membrane $\mathrm{K}^{+}$- permeable channels. Plant Physiology 141: 1653- 1665.

Sohan D, Jasoni R, Zajicek J, 1999. Plant-water relations of $\mathrm{NaCl}$ and calcium-treated sunflower plants. Envriron. Exp. Bot. 42: 105111. 
Song JY, Rose JH, 2008. The role and regulation of Trxl, a cytosolic thioredoxin in Schizosaccharomyces pombe. The Journal of Microbiology 46: 408-414.

Wouyou A, Gandonou CB, Assogba Komlan F, Montcho D, Zanklan SA, Lutts S, Gnancadja SL, 2017. Salinity Resistance of Five Amaranth (Amaranthus cruentus) Cultivars at Young Plants Stage, International Journal of Plant and Soil Science 14(3): 1-13.

Wouyou A, Prodjinoto H, Zanklan AS, Vanpee B, Lutts S, Gandonou CB, 2019. Implication of ions and organic solutes accumulation in amaranth (Amaranthus cruentus L.) salinity resistance, American Journal of Plant Sciences $10: 2335$ 2353. 